Aim of the study: To retrospectively assess treatment outcomes among patients treated for salivary gland cancers at our institution to determine which of the three most common treatment approaches - elective neck dissection (END), elective neck irradiation (ENI), or observation - provide the best results.

Material and methods: A total of 122 patients were identified who had undergone primary surgery for SGC followed by END, ENI, or observation. The patients were classified into three groups according to the treatment approach used to manage the neck: END, ENI, or observation. The main outcome measures were disease-free survival (DFS) and overall survival (OS). We also sought to identify the risk factors potentially associated with neck metastasis and treatment failure.

Results: 106 patients met all inclusion criteria. Of these 106 patients, 27 (25.7\%) underwent END, 17 (16.0\%) underwent ENI, and 62 (58.5\%) observation. There were no statistically significant differences between the three groups in any of the following variables: advanced age (> 70); presence of locally advanced disease (T3 or T4); perineural invasion; lymphovascular invasion; and primary tumour location. Treatment failure was higher (non-significantly) in the END group (25.9\%) vs. the observation (21.0\%) and ENI (11.8\%) groups. No differences (Kaplan-Meir curves) were observed among the three groups in terms of DFS or OS.

Conclusions: Our results show that elective neck dissection does not appear to provide any benefit to patients treated for malignant salivary gland cancer. Importantly, these findings contradict most of the currently available research. However, due to methodological differences among the available studies, our findings cannot be compared directly to other studies.

Key words: head, neck, salivary glands, elective neck dissection, radiotherapy, surveillance.

Contemp Oncol (Pozn) 2019; 23 (3): 169-173 DOI: https://doi.org/10.5114/wo.2019.89245

\section{Management of clinically negative neck in salivary gland cancers - elective neck dissection, irradiation, or surveillance?}

\author{
Mateusz Szewczyk ${ }^{1}$, Paweł Golusiński², Jakub Pazdrowski ${ }^{1}$, \\ Piotr Pieńkowski ${ }^{1}$, Wojciech J. Golusiński ${ }^{1}$
}

${ }^{1}$ Department of Head and Neck Surgery, The Greater Poland Cancer Centre, Poznan University of Medical Sciences, Poznan, Poland

${ }^{2}$ Department of Otolaryngology and Maxillofacial Surgery, University of Zielona Gora, Zielona Gora, Poland

\section{Introduction}

Salivary gland cancer (SGC) is a relatively rare cancer, accounting for approximately $6 \%$ of all head and neck cancers [1]. According to the World Health Organisation (WHO), there are 24 different SGC subtypes with heterogenous pathology and origin, which explains why treatment is so challenging in these tumours [2]. SGC is typically divided into major and minor SGC. The most common subtypes of major SGC are parotid gland and submandibular SGC. The minor subtypes generally arise from glands located in the mucosa across the head and neck region, most commonly in the oral cavity. Radical resection, with or without adjuvant radiotherapy in patients with high-risk factors, is the mainstay treatment for primary SGC [3]. However, management of the neck is generally more complex. In patients with node-positive neck disease, the general consensus is therapeutic or modified radical neck dissection with excision of nodal levels I-V. By contrast, in clinically node-negative patients, the optimal approach is unclear and controversial. While many authors recommend elective neck dissection (END) in all patients with malignant SGC, others recommend elective neck irradiation (ENI) or observation [4-7]. Therefore, at present, the optimal therapeutic approach remains uncertain, mainly because most of the available data on neck management come from small, retrospective, single-centre studies with ambiguous outcomes [4, 8-10]. Moreover, clinical guidelines are not currently available to guide treatment.

Given the scarce data regarding the optimal approach to neck management in patients with SGC, it is evident that more data are needed to help clarify this issue. In this context, the aim of the present study was to retrospectively assess treatment outcomes among patients treated for SGC at our institution, to determine which of the three most common treatment approaches - END, ENI, or observation - provides the best results.

\section{Material and methods}

This was a retrospective analysis of patients who underwent surgery for SGC at our institution between the years 2007 and 2017. All patients underwent primary surgery followed by either END, ENI, or observation.

The inclusion criteria were as follows: 1) diagnosis of SGC; 2) surgical resection of the primary tumour: 3 ) negative neck lymph nodes (NO) - each patient had ultrasonography examination of neck + computed tomography (CT)/magnetic resonance imaging (MRI). Whenever there was a suspicious lymph node in any examination a fine needle aspiration (FNA) of the lesion was made to confirm/exclude node metastasis; and 4) $\geq 6$ months of fol- 
low-up (except for cases in which treatment failure occurred earlier). Exclusion criteria were diagnosis of a second primary tumour and recurrent disease.

From our institutional database, we identified a total of 122 patients who had undergone primary surgery for SGC followed by END, ENI, or observation. The patients were classified into three groups according to the treatment approach used to manage the neck: END, ENI, or observation. The main outcome measures were disease-free survival (DFS) and overall survival (OS). We also sought to identify the risk factors potentially associated with neck metastasis and treatment failure.

All patients were evaluated both clinically and radiologically (ultrasound, CT, and/or MRI). The following clinical data were obtained and recorded: age at diagnosis; sex; local disease stage according to the 2009 American Joint Committee on Cancer criteria; tumour grade; pathologic subtype; tumour location; perineural invasion (PNI); lymphovascular invasion (LVI); and type of treatment failure (regional, distant or combined). Cases of isolated local recurrence were not considered in the statistical analysis.

Prior to treatment, all patients with NO neck disease were presented at a multidisciplinary tumour board, which evaluated a wide range of factors before making the treatment decision. The following variables were considered in deciding whether to propose END: the presence of locally advanced disease; high tumour grade; high-risk histological type (i.e. salivary duct carcinoma, adenocarcinoma not otherwise specified [NOS], or mucoepidermoid carcino$\mathrm{ma}$ ), and patient preferences. When the full histological report was available, those same factors were also considered when deciding to perform ENI. Finally, patients who were not considered suitable due to the absence of highrisk factors for END or ENI, or who refused either of those procedures, were assigned to the observation group.

\section{Statistical analysis}

Statistical analysis was performed using the Statistica software package, v. 12 (StatSoft Inc., Tulsa, OK, USA). Outcome measures included disease-free survival (DFS) and overall survival (OS). DFS and OS were calculated from the date of surgery until the date of recurrence or last follow-up visit (DFS) or until death or last follow-up visit (OS). Kaplan-Meier methods were used to estimate survival outcomes. The log-rank test was used to compare survival curves.

The chi-square test was used to determine if clinical variables including age, advanced $T$ stage, high grade of tumour, PNI, LVI, location of the tumour, and histopathologic subtype differed between neck management groups: elective neck dissection, elective neck irradiation, and observation. A value of $p<0.05$ was considered statistically significant.

\section{Results}

\section{Patient characteristics}

Of the 122 candidates screened for possible inclusion in this study, 106 met all inclusion criteria and were included. Of these 106 patients, 27 (25.7\%) underwent END, 17 (16.0\%) underwent ENI, and 62 (58.5\%) observation. Mean (standard deviation - SD) follow-up was 31 months (28.8).

There were no statistically significant differences (Table 1) between the three groups in any of the following variables: advanced age (> 70 years); presence of locally-advanced disease (T3 or T4); perineural invasion; lymphovascular invasion; and primary tumour location. The only significant difference between the groups was a higher proportion of patients in the END group with high-grade tumours: $55.6 \%$ vs. $20.8 \%$ and $33.3 \%$, respectively, in the observation and END groups ( $p=0.007$ ). Treatment failure was higher (non-significantly) in the END group (25.9\%) vs. the observation (21.0\%) and ENI (11.8\%) groups (Table 1). No differences (Kaplan-Meir curves) were observed among the three groups in terms of DFS or OS (Figs. 1 and 2).

The most commonly dissected nodal levels were level 2 and 3, with a median of 16 nodes per patient (range 8-33). A total of eight pathologically positive lymph nodes were detected in three of the patients in the END group, distributed as follows: $n=1$ node in one patient; $n=2$ in one patient; $n=5$ in one patient.

Table 1. Examined variables in treatment groups

\begin{tabular}{|c|c|c|c|c|c|c|c|c|c|}
\hline $\begin{array}{l}\text { Treatment } \\
\text { group } \\
(n=106)\end{array}$ & $\begin{array}{l}\text { Patients/ } \\
\text { group }\end{array}$ & Age $>70$ & Stage T3/4 & High grade & PNI & LVI & $\begin{array}{l}\text { Gland location: } \\
\text { a) parotid } \\
\text { b) submandibular } \\
\text { c) minor }\end{array}$ & $\begin{array}{l}\text { Most common } \\
\text { pathologic } \\
\text { subtype }\end{array}$ & $\begin{array}{l}\text { Treatment } \\
\text { failure }\end{array}$ \\
\hline END, $n(\%)^{\star}$ & 27 & $5(18.5)$ & $9(33.3)$ & $12 / 22(54.6)$ & $9(33.3)$ & $3(11.1)$ & $\begin{array}{l}\text { a) } 15(55.6) \\
\text { b) } 9(33.3) \\
\text { c) } 3(11.1)\end{array}$ & CaExPA 5 (18.5) & $7(25.9)$ \\
\hline OBS, $n(\%)^{*}$ & 62 & $14 / 61(23.0)$ & $17 / 61(27.9)$ & $10 / 48(20.8)$ & 11/61 (18.6) & 6/61 (9.7) & $\begin{array}{l}\text { a) } 38(62.3) \\
\text { b) } 7(11.5) \\
\text { c) } 17(26.2)\end{array}$ & ACC 16/61 (26.2) & $13 / 61(21.0)$ \\
\hline ENI, $n(\%)^{*}$ & 18 & $5(27.8)$ & $6(35.3)$ & 5/15 (33.3) & $6(33.3)$ & $3(17.7)$ & $\begin{array}{l}\text { a) } 9(50.0) \\
\text { b) } 4(22.2) \\
\text { c) } 5(27.8)\end{array}$ & ACC 18 (38.9) & $2(11.8)$ \\
\hline$p$-value & & NS & NS & 0.007 & NS & NS & NS & NA & NS \\
\hline
\end{tabular}

END - elective neck dissection, OBS - observation group, ENI - elective neck irradiation, PNI - perineural invasion, LVI - lymphovascular invasion, CaExPA carcinoma ex-pleomorphic adenoma, ACC - adenoid cystic carcinoma, NS - not significant, NA - not applicable; "all percentages of based on the number of patients in each group unless otherwise indicated 

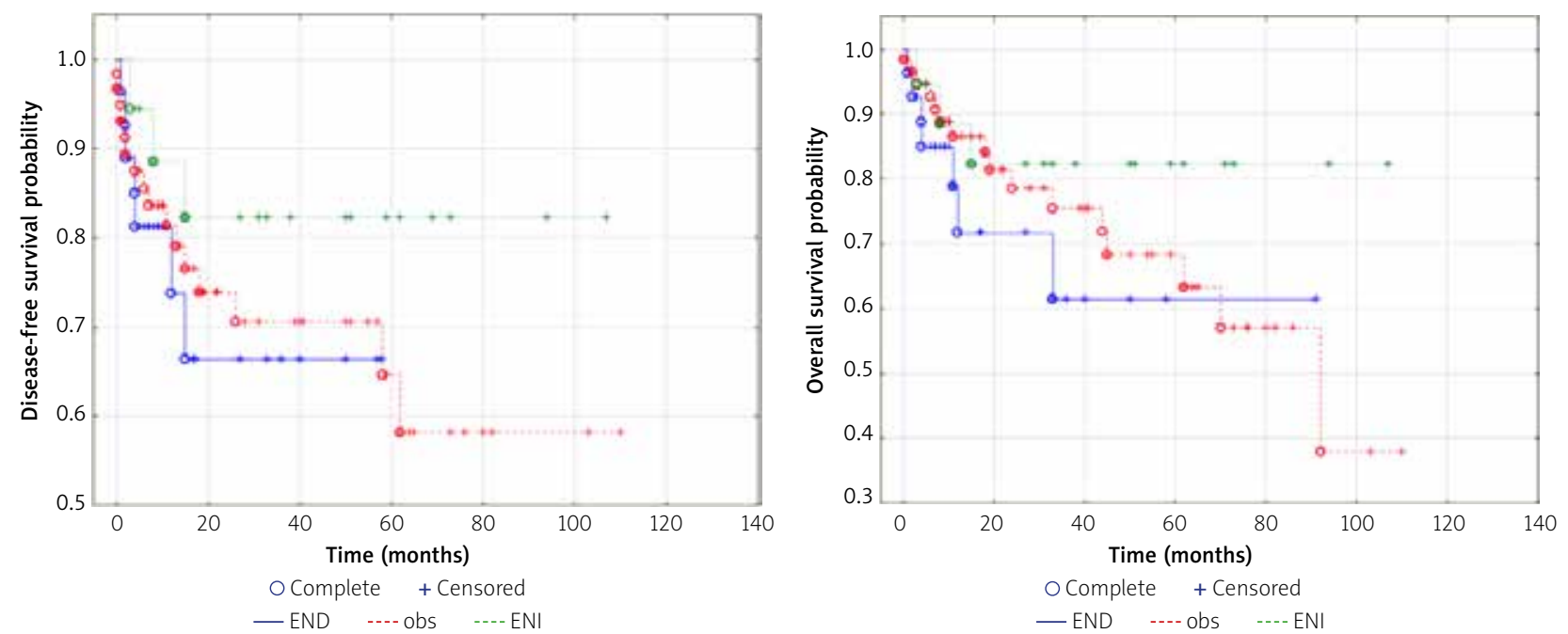

Fig. 1. Disease-free survival by treatment group

Fig. 2. Overall survival by treatment group

END - elective neck dissection, obs - observation group, ENI - elective neck irradiation

\section{Discussion}

In the present study, we compared treatment outcomes among patients who underwent primary surgery for SGC to determine the role of the specific approach to neck management (END, ENI, or observation) on OS or DFS. We also wanted to determine the risk factors associated with neck metastasis and treatment failure. At baseline, the only significant difference between the three groups was a higher percentage of patients in the END group with high-grade disease (55.6\% vs. $20.8 \%$ and $33.3 \%$, respectively) in the observation and END groups $(p=0.007)$. We found a non-significant trend towards a higher treatment failure rate in the END group $-25.9 \%$ vs. $21.0 \%$ and $11.8 \%$ in the observation and ENI groups (Table 1). However, we found no significant between-group differences in 10-year DFS or OS rates.

Due to the relative rarity of SGC and the lack of randomised controlled trials, treatment protocols - including those for neck management in these patients - are based primarily on retrospective single-centre studies. In the early 1990s, Armstrong et al. [5] reported the results of a large study involving 474 patients with major SGC, most of whom $(n=407)$ had clinically-negative neck nodes. Of these 407 patients with NO disease, 90 underwent END, which revealed occult disease in 34 cases (38\%). A logistic regression analysis showed that tumour grade and the presence of locally advanced disease were both significant predictors of occult disease. Neck recurrence was detected in $8 \%$ of patients in the observation group (who did not undergo either END or ENI) vs. $14 \%$ of the patients who had undergone END. The authors hypothesised that this difference could be attributed to the fact that the patients with the highest risk disease underwent END, and thus a higher recurrence rate should be expected in that group. Of the patients with occult metastasis, treatment failure was observed in $29 \%$ of the patients who did not receive radiotherapy vs. $0 \%$ of those who did, leading the authors to recommend the routine use of adjuvant radiotherapy in patients with proven occult neck metastases [5]. Consistent with the findings reported by Armstrong et al., none of the three patients in our cohort with occult neck disease - all of who received adjuvant RT - developed a recurrence.

Armstrong et al. did not separately report the results of the patients who underwent ENI, so we cannot directly compare our findings for the ENI group with the results of that study. However, in a more recent study [7] involving 83 patients with parotid gland cancer (no other subtypes were included), Zbären et al. subdivided the patients into two treatment groups: END $(n=41)$ and observation $(n=42)$. At baseline, there were no significant between-group differences in any of the clinical, sociodemographic, or pathological variables. Of the 41 patients who underwent neck dissection, eight (20\%) had occult neck disease, and six of these patients later received adjuvant RT. Treatment failure occurred in $12 \%$ and $26 \%$, respectively, of the END and observation groups. Although some of the patients included in the observation group received ENI, the authors did not separately report the results for that group. However, they did report that two of the patients who underwent ENI developed a recurrence. In that study, DFS was better (non-significantly) in the END group, without significant between-group differences in OS. Those findings led the authors to conclude that END is preferable in all parotid gland cancers. However, our results appear to contradict that conclusion, as we did not observe any statistical benefit for END. Due to the methodological differences (i.e. patient stratification and SGC subtypes) between that study and ours, it is not possible to directly compare results. However, it is worth noting that tumour size and tumour grade had no influence on the presence of neck metastasis: of the eight patients in their cohort with occult neck disease, three had low-grade tumours and four had stage T2 cancer [7].

Chen et al. [11] examined 251 patients with SGC, who underwent primary surgery. Of those, 131 patients (52\%) underwent ENI, while the other 120 (48\%) underwent ob- 
servation alone. None of the patients in that study received neck dissection. There were no between-group differences in any clinical or pathological characteristics. Importantly, neck irradiation had no impact on OS (10-year OS: $58 \%$ in the observation group vs. $51 \%$ in the ENI group), but there was a significant difference in neck recurrence rates (26\% vs. $0 \%$, respectively). The authors concluded that patients with high T stage and high-risk histological features could be safely treated with ENI, potentially obviating the need for END. However, it is important to keep in mind that the exact tumour histology is not always available prior to surgery, and therefore the use of END is not always planned in tumours whose histological characteristics place them originally at low risk of failure. In our study, all of the patients in the END group underwent preoperative fine-needle aspiration cytology, and in less than $30 \%$ of those cases $(8 / 27)$ the result was suggestive of a high-risk histological subtype. Nonetheless, positive surgical margins are relatively common in SGC (ranging from $32 \%$ to $64.7 \%$ ), and thus postoperative radiotherapy to the primary tumour is often warranted; moreover, including the neck within the radiotherapy field typically induces few or no additional side effects.

Herman et al. [12] conducted a single-centre study involving 59 patients with high-grade cNO SGC. Of these 59 cases, 41 underwent END and 18 ENI. However, the authors did not describe how the patients were stratified. Occult neck metastasis was found in $44 \%$ of patients in the END group. Neck recurrence was observed in $10 \%$ of the patients who underwent neck dissection vs. $0 \%$ in the ENI group. Those results support the use of elective irradiation, even in patients with high-grade tumours; however, as those authors noted, the patients in the END group were more likely to present with locally-advanced disease and high-grade mucoepidermoid carcinoma, which could have influenced their results [12].

Reviews carried out by Wang et al. and Green et al. $[13,14]$ attempted to identify the specific features of SGC, which could indicate the need for END. Among the reviewed studies, the two most common indicators for END were high-grade disease and locally advanced disease. Other studies suggest that the primary tumour site is also a key factor, with some authors mentioned in the review by Green et al. stating that all patients with SGC - except for those with oral cavity tumours - should undergo prophylactic neck dissection [14]. The optimal management of these tumours remains controversial. As Medina et al. [15] concluded in their review, given the contradictory data in the literature, the debate remains open. Medina et al. discuss how beliefs about the optimal management of the neck have changed in recent decades [15]. In this regard, regrettably, the findings of our study cannot resolve the ongoing debate. In fact, they only serve to further confirm the need to clarify this question.

\section{Study strengths and limitations}

An important limitation of our study is the diversity of tumour locations and types included in our cohort. In addition, this was a retrospective study, and thus potential se- lection bias cannot be excluded. Finally, we did not use any strict criteria with regard to the treatment decision (END, ENI, or observation) because this decision was made on a case by case basis and also took into account patient preferences. The strengths of our study are the single-centre design and the large number of patients, all of whom underwent the same surgical procedure.

\section{Conclusions}

Our results show that elective neck dissection for NO neck does not appear to provide any benefit to patients treated for malignant salivary gland cancer. Importantly, these findings contradict most of the currently available research. However, due to methodological differences among the available studies, our findings cannot be compared directly to other studies. Therefore, a prospective multicentre study is needed to determine the optimal management of the NO neck in patients with SGC.

\section{Acknowledgements}

The Greater Poland Cancer Centre Grant 15/2015(107) provided financial support for the conduct of the research and preparation of the article mainly in the collection and analysis of data.

\section{The authors declare no conflict of interest.}

\section{References}

1. Hosni A, Huang SH, Goldstein D, et al. Outcomes and prognostic factors for major salivary gland carcinoma following postoperative radiotherapy. Oral Oncol 2016; 54: 75-80.

2. Laurie SA, Licitra L. Systemic Therapy in the Palliative Management of Advanced Salivary Gland Cancers. J Clin Oncol 2006; 24 : 2673-2678.

3. Lombardi D, McGurk M, Vander Poorten V, et al. Surgical treatment of salivary malignant tumors. Oral Oncol 2017; 65: 102-113.

4. Ali S, Palmer FL, DiLorenzo M, Shah JP, Patel SG, Ganly I. Treatment of the Neck in Carcinoma of the Parotid Gland. Ann Surg Oncol 2014; 21: 3042-3048.

5. Armstrong JG, Harrison L, Thaler H, Friedlander-Klar H. The Indications for Elective Treatment of the Neck in Cancer of the Mayor Salivary Glands. Cancer 1992; 92: 615-619.

6. Kawata R, Koutetsu L, Yoshimura K, Nishikawa S, Takenaka H. Indication for elective neck dissection for NO carcinoma of the parotid gland: a single institution's 20-year experience. Acta Otolaryngol (Stockh) 2010; 130: 286-292.

7. Zbären P, Schüpbach J, Nuyens M, Stauffer E. Elective neck dissec tion versus observation in primary parotid carcinoma. Otolaryngol-Head Neck Surg 2005; 132: 387-391.

8. Ettl T, Gosau M, Brockhoff G, et al. Predictors of cervical lymph node metastasis in salivary gland cancer: Lymph Nodes in Salivary Gland Cancer. Head Neck 2014; 36: 517-523.

9. Lau VH, Aouad R, Farwell DG, Donald PJ, Chen AM. Patterns of nodal involvement for clinically NO salivary gland carcinoma: Refining the role of elective neck irradiation: Nodal involvement for salivary carcinoma. Head Neck 2014: 36: 1435-1439.

10. Nobis C-P, Rohleder NH, Wolff K-D, Wagenpfeil S, Scherer EQ, Kesting MR. Head and Neck Salivary Gland Carcinomas - Elective Neck Dissection, Yes or No? J Oral Maxillofac Surg 2014; 72: 205-210.

11. Chen AM, Garcia J, Lee NY, Bucci MK, Eisele DW. Patterns of nodal relapse after surgery and postoperative radiation therapy for carcinomas of the major and minor salivary glands: What is the role of elective neck irradiation? Int J Radiat Oncol 2007; 67: 988-994. 
12. Herman MP, Werning JW, Morris CG, Kirwan JM, Amdur RJ, Mendenhall WM. Elective neck management for high-grade salivary gland carcinoma. Am J Otolaryngol 2013; 34: 205-208.

13. Wang Y-L, Li D-S, Gan H-L, et al. Predictive index for lymph node management of major salivary gland cancer. Laryngoscope 2012 122: 1497-1506.

14. Green B, Rahimi S, Brennan PA. Current management of the neck in salivary gland carcinomas. J Oral Pathol Med 2017; 46: 161-166.

15. Medina J, Zbären P, Bradley PJ. Management of Regional Metastases of Malignant Salivary Gland Neoplasms. In: Salivary Gland Neoplasms, vol. 78, Bradley PJ, Eisele D (eds.). Karger, Basel 2016: $132-140$.

\section{Address for correspondence}

\section{Mateusz Szewczyk}

Department of Head and Neck Surgery

The Greater Poland Cancer Centre

Poznan University of Medical Sciences

15 Garbary St.

61-866 Poznan, Poland

e-mail: mateuszszewczyk@yahoo.pl

Submitted: 30.04 .2019

Accepted: 16.06.2019 